\title{
Tetradecanoylphorbol Acetate
}

National Cancer Institute

\section{Source}

National Cancer Institute. Tetradecanoylphorbol Acetate. NCI Thesaurus. Code C866.

A phorbol ester with potential antineoplastic effects. Tetradecanoylphorbol acetate (TPA) induces maturation and differentiation of hematopoietic cell lines, including leukemic cells. This agent may induce gene expression and protein kinase C (PKC) activity. In addition to potential antineoplastic effects, TPA may exhibit tumor promoting activity. (NCI04) 